\title{
Sulla rappresentazione delle funzioni di variabile bicomplessa totalmente derivabili.
}

\author{
Memoria di Nrcoló Spamprnato (a Catania).
}

Snnto. - Si studiano le superficie e le trasfornazioni caratteristiche dell' $\mathrm{S}_{4}$ complesso. immagini dei legani e delle trasformazioni su due variabili bicomplesse ottenute con fun. zioni totalmente derivabili.

In una importante Memoria ( ${ }^{\dagger}$ ), Bexramino SEgRE ha studiato geometricamente i legami analitici e le trasformazioni analitiche su due variabili complesse, che danno laogo, nell' $S_{4}$ rappresentativo di queste coppie di variabili, alle superficie caratteristiche e alle trasformazioni pseudoconformi.

L'idea semplificatrice che il SEGRE ha avuto è stata quella di non restringersi a considerare nell' $S_{4}$ soltanto elementi reali, ma anche quelli immaginari e ha ricordato, in proposito, che tale estensione nell' $S_{4}$ rappresentativo è stata fatta da CoRRAdo SEGre, quando ha introdotto nel piano complesso i punti bicomplessi $\left({ }^{2}\right)$, ed equivale a prolungare nel corpo complesso le componenti reali delle coppie di variabili complesse, ovvero a considerare coppie di variabili bicomplesse anzichè coppie di variabili complesse ( $\left.{ }^{3}\right)$.

Si osservi ora che se la variabile complessa $y$ è funzione olomorfa della variabile complessa $x$, prolungarido le componenti reali di $x$ e $y$ nel corpo complesso, cioè prolungando le variabili $x$ e $y$ nell'algebra dei numeri bicomplessi, si ottiene la variabile bicomplessa y funzione olomorfa secondo

(1) B. SEgre. Questioni geometriche legate alla teoria delle funzioni di due variabili complesse ["Rendiconti del Seminario Matematico della R. Università di Roma", Vol. VII. Parte II, 1980-31-X, pp. 59-107].

(2) C. SEGRE, Sulle rappresentazioni reali delle forme complesse e gli enti iperalgebrici [" Mathematische Annalen ", XL Band (1892), pp. 413-467].

(3) Si veda in proposito la mia nota: I punti bicomplessi e le varietà iperalgebriche del Segre ["Esercitazioni Matematiche», Vol. VIII, pp. 67-76, "Circolo Matematico di Catania s; 1935-XIII]. 
Scorqa Dragoni (H) della variabile bicomplessa $x$. Ne segue ehe, corrispon. dentemente, i legami analitici e le trasformazioni analitiche su due variabili complesse si prolungano nei legami e nelle trasformazioni su due variabili bicomplesse ottenute con funzioni olomorfe secondo Scorza Dragon. Pertanto questi legami e queste trasformazioni danno luogo, nell' $S_{4}$ complesso rappresentativo, alle superficie $e$ alle trasformazioni che generalizzano le super* ficie caratteristiche e le trasformazioni pseudoconformi dell' $S_{4}$ reale, e che chiameremo superficie caratteristiche e trasformazioni caratteristiche dell' $S_{4}$ complesso.

In una nota in corso di stampa nei « Rendiconti della R. Accademia dei Lincei $\gg$ ho dimostrato che le funzioni di variabile bicomplessa olomorfe se. condo Sconza Dragont coincidono con le funzioni, nell'algebra dei numeri bicomplessi, totalmente derivabili ovvero con le funzioni a derivata caratteristica unica. Da tale nota risulta manifesta la convenienza di usare nello stadio di dette funzioni il sistema di unità che mette in evidenza la riducicibilità dell' algebra dei numeri bicomplessi.

A riguardo si osservi che il SEVERI nell'applicare il metodo che chiama del passaggio dal reale al complesso $\left({ }^{5}\right)$ per la risoluzione generale del problema di DIRICHLET per le funzioni biarmoniche, eseguisce la seguente tra* sformazione sulle due coppie di variabili complesse $\left(x_{1}, x_{2}\right),(x, \bar{x})$ :

$$
\left\{\begin{array} { l l } 
{ x = x _ { 1 } + i x _ { 2 } } \\
{ \overline { x } = x _ { 1 } - i x _ { 2 } }
\end{array} \quad \left\{\begin{array}{l}
x_{1}=(x+\bar{x}) / 2 \\
x_{2}=(x-\bar{x}) / 2
\end{array}\right.\right.
$$

Ebbene, le (1) sono le relazioni che intercedono fra le coordinate $x_{1}, x_{2}$ e $x, \bar{x}$ di uno stesso numero bicomplesso nei due sistemi di unità $u, v \mathrm{e}$ $v_{1}, v_{2}$ con le seguenti tabelle di moltiplicazione:

$$
\begin{aligned}
& u^{2}=u, \quad u v=v u=v, . v^{2}=-u \\
& v_{1}^{2}=v_{1}, \quad v_{1} v_{2}=v_{2} v_{1}=0, \quad v_{2}^{2}=v_{2} .
\end{aligned}
$$

Sicchè quando dalle variabili complesse $x_{1}$ e $x_{2}$ si passa alle variabili complesse $x$ e $\vec{x}$, si viene ad esprimere la variabile bicomplessa $z=x_{1} u+x_{2}$ : (prolungamento della variabile complessa $x_{1}+i x_{2}$, corrispondente al prolunga. mento dei numeri reali $x_{1}$ e $x_{2}$ nel corpo complessol nella forma $z=x v_{1}+\bar{x} v_{z}$,

(1) G. SoORzA Dragont, Sulle funzioni olomorfe di wna variabile bicomplessa [" Reale Accademia d'Italia ", Memorie della Classe di Scienze, Vol. V, pp. 597-665].

(5) F. Severi, Risolueione generale del problema di DIRIChLet per le funzioni bian. moniche ["Rendiconti della R. Accademia dei Lincei ", Vol. XIII, 1981, pp. 795-804]. 
cioè nel sistema di unità $v_{1}, v_{2}$ che è quello che mette in evidenza la riducibilità dell' algebra dei numeri bicomplessi.

Le superiori osservazioni spiegano perchè nello studio che faccio in questa Nota intorno alle superficie e alle trasformazioni caratteristiche dell' $S_{4}$ complesso, uso sistematicamente le unità $v_{1}$ e $v_{2}$, ottenendo notevoli semplificazioni.

\section{$\$ I$.}

1. Le superficie dell' $s_{4}$ complesso immagini delle funzioni di una variabile bicomplessa totalmente derivabili. - Sia $C^{\prime}$ l'algebra dei numeri bicomplessi

$$
x=x_{1} v_{1}+x_{2} v_{2}
$$

con $v_{1}, v_{2}$ unità soddisfacenti alle condizioni

$$
v_{1}^{2}=v_{1}, \quad v_{1} v_{2}=v_{2} v_{1}=0, \quad v_{2}^{2}=v_{2}
$$

e $x_{1}, x_{2}$ numeri complessi. In corrispondenza ad una coppia di numeri bicomplessi $(x, y)$, posto per $y$

$$
y=y_{1} v_{1}+y_{2} v_{2}
$$

consideriamo in un $S_{+}$complesso, dove si suppone fissato un sistema di coordinate non omogene, cartesiane, il punto $P\left(x_{1}, x_{2}, y_{1}, y_{2}\right)$, che si dirù immagine della coppia di numeri bicomplessi $(x, y)$.

Cio posto, consideriamo una funzione $y(x)$ della variabile bicomplessa $x$, totalmente derivabile, cioè olomorfa secondo Scorza DRAGONI, e quindi del tipo

$$
y(x)=y_{1}\left(x_{1} w_{1}+y_{2}\left(x_{2}\right) w_{2}\right.
$$

con $y_{1}\left(x_{1}\right)$ e $y_{2}\left(x_{2}\right)$ funzioni olomorte delle variabili complesse $x_{1}$ e $x_{2}$ rispettivamente.

Mentre $x$ varia in un campo contenuto nel campo di esistenza della $y(x)$, il punto $P$ dell' $S_{4}$ complesso immagine della coppia di numeri bicomplessi $(x, y(x))$ descriverà un pezzo della superfieie, di equazioni

$$
y_{1}=y_{1}\left(x_{1}\right), \quad y_{2}=y_{2}\left(x_{2}\right)
$$

che si dirà la superficie caratteristica dell' $\mathrm{S}_{4}$ complesso, immagine della funzione bicomplessa $y(x)$ totalmente derivabile.

In particolare, come immagini delle funzioni lineari totalmente derivabili

$$
y(x)=\left(a x_{1}+b\right) v_{1}+\left(c x_{2}+d\right) v_{2}
$$


avremo i piani caratteristici dell' $S_{4}$ complesso di equazioni

$$
y_{1}=a: x_{1}+b, \quad y_{2}=c x_{z}+d \text {. }
$$

2. Piani caratteristici dell' $\boldsymbol{S}_{4}$ complesso. - Indichiamo con $r^{\prime}$ e $r^{\prime \prime}$ le refte improprie dei piani coordinati $\rho_{1}\left(x_{1}, y_{1}\right)$ e $\rho_{2}\left(x_{2}, y_{2}\right)$. La retta impropria del piano caratteristico di equazioni (5) s'appoggia alle rette sghembe $r^{\prime}$ e $r^{\prime \prime}$ e precisamente nei punti impropri delle due rette tracce, in detti piani, dei due iperpiani che le (5) rappresentano separatamente. Le stesse equazioni rappresentano nei due piani $\left(x_{1}, y_{1}\right)$ e $\left(x_{2}, y_{2}\right)$ tali tracce. Evidentemente il punto di appoggio in $r^{\prime}\left(r^{\prime \prime}\right)$ è distinto dal punto improprio $Y_{1}\left(Y_{2}\right)$ di $y_{1}\left(y_{2}\right)$. Viceversa ogni piano dell' $S_{4}$, appoggiato ad $r^{\prime} e$ ad $r^{\prime \prime}$ in punti distinti da $Y_{1}$ e $Y_{2}$, ha le equazioni del tipo. (5) e quindi rappresenta una funzione lineare bicomplessa totalmente derivabile, cioè è un piano caratteristico. Si osservi che le eccezioni presentate da $Y_{1}$ e $Y_{2}$ provengono dal fatto che si considerano le funzioni $y(x)$ totalmente derivabili seritte sotto la forma esplicita [1]. Se alla [1] si sostituisce l' equazione

$$
H_{1}\left(x_{1}, y_{1}\right) v_{1}+H_{2}\left(x_{2}, y_{2}\right) v_{2}=0
$$

con $H_{1}$ e $H_{2}$ simboli di legami analitici fra $x_{1}$ e $y_{1}$ efra $x_{2}$ e $y_{2}$, e quindi alla (4) si sostituisce l'equazione

$$
\left(a_{1} x_{1}+b_{1} y_{1}+c_{1}\right) v_{1}+\left(a_{2} x_{2}+b_{2} y_{2}+c_{2}\right) v_{2}=0
$$

i casi di eccezione, che corrispondono ai valori $b_{1}=0$ e $b_{2}=0$ vengono ad essere eliminati. Abbiamo pertanto che:

I) I piani caratteristici dell' $\mathrm{S}_{4}$ complesso, cioè $i$ piani immagini delle funzioni lineari della variabile bicomplessa $\mathrm{x}$, derivabili totalmente, ovvero olomorfe secondo SCORzA DRAGoNI, sono tulti e soli $i$ piani appoggiali alle rette sghembe $\mathrm{r}^{\prime}$ e $\mathrm{r}^{\prime \prime}\left({ }^{\mathrm{a}}\right)$.

3. Superficie caratteristiche dell' $\boldsymbol{S}_{4}$ complesso. - Consideriamo nuovamente la superficie caratteristica $F$ di equazioni [2], immagine della funzione $y(x)$, totalmente derivabile, data dalla [1]. La prima (seconda) delle equazioni [2] rappresenta nel piano coordinato $\rho_{1}\left(x_{1}, y_{1}\right)\left(o_{2}\left(x_{2}, y_{2}\right)\right)$ un pezzo di

(r) Si noti che le rette $r^{\prime}$ e $r^{\prime \prime}$, rette improprie dei piani coordinati $p_{1}$ e $p_{2}$, coincidono con le rette $r^{\prime}$ e $r^{\prime \prime}$, assi della congruenza $K$ considerata dal Skgre nella sua memoria eitata in (1), prefazione e $\mathrm{n}^{\circ} 1$. Nel sistema di coordinate usato dal SEGRE (che conrisponde alle unità $\mathrm{n}$ e v) le rette $r^{\prime}$ e $r^{\prime \prime}$ sono immaginarie coniugate. Nel sistema di coordinate considerato in questa nota (che corrisponde alle unito $\mathrm{v}_{1}$ e $\mathrm{v}_{2}$ ) le rette $r^{\prime}$ e $r^{\prime \prime}$ sono reali. 
curva analitica $f_{1}\left(f_{2}\right)$. Se $P_{1}\left(P_{2}\right)$ è un punto qualunque di $f_{1}\left(f_{2}\right)$ i piani $P_{1} r^{\prime \prime}$ e $P_{2} r^{\prime}$ si secano in un punto $P$ di $F$. La $F$ è pertanto intersezione di due pezzi di $S_{1}$-coni, ehe si ottengono proiettando $f_{1}$ da $r^{\prime \prime}$ e $f_{2}$ da $r^{\prime}$. Gl' $\infty^{1}$ piani proiettanti i punti di $f_{1}$ da $r^{\prime \prime}$ secano $F$ in un sistema, di dimensione complessa 1, di curve ciascuna delle quali $\dot{e}$ proiettata da $r^{\prime}$ sul piano $P_{2}$ nella curva $f_{2}$.

Un secondo sistema di curve, di dimensione complessa 1, si ottiene secando la $F^{\prime}$ con i piani proiettanti i punti di $f_{2}$ da $r^{\prime}$, Ciascuna curva di questo secondo sistema è proiettata da $r^{\prime \prime}$ su $p_{1}$ nella curva $f_{1}$. La proiezione della $F$ da $r^{\prime \prime}\left(r^{\prime}\right)$ su $\rho_{i}\left(\rho_{2}\right)$ non è, pertanto, un pezzo di piano a 2 dimensioni, ma un pezzo di curva $f_{1}\left(f_{2}\right)$. Viceversa se si ha nello spazio complesso $S_{4}$ una superficie (o pezzo di superficie) $F$ che è proiettata da $r^{\prime}\left(r^{\prime \prime}\right)$ su $\rho_{1}\left(\rho_{z}\right)$ in un pezzo di curva analitica $f_{1}\left(f_{2}\right)$, se questa è immagine di una funzione olomorfa $y_{1}\left(x_{1}\right)\left(y_{2}\left(x_{2}\right)\right)$, la $F$ è caratteristica e precisamente è l'immagine della funzione bicomplessa totalmente derivabile avente per coefficienti di $v_{1}$ e $v_{z}$ le due funzioni $y_{1}\left(x_{1}\right)$ e $y_{2}\left(x_{2}\right)$. Concludendo si ha:

II) Le superficie caratteristiche dell' $\mathrm{S}_{4}$ complesso, ciò̀ immagini delle funzioni di variabile bicomplessa totalmente derivabili, o olomorfe secondo SCoRza DragonI, sono tutte e sole le superficie dell' $\mathbb{S}_{*}$ complesso proiettate da $\mathrm{r}^{\prime}$ e $\mathrm{r}^{\prime \prime}$, rispellivamente sui piani coordinati $\rho_{2} e \rho_{1}$ in curve analitiche immagini di funzioni olomorfe $\mathrm{y}_{1}\left(\mathrm{x}_{1}\right), \mathrm{y}_{2}\left(\mathrm{x}_{2}\right)$ delle variabili complesse $\mathrm{x}_{1}$ e $\mathrm{x}_{2}$ rispettivamente.

4. Curve e piani seminulli dell' $\boldsymbol{S}_{4}$ complesso. - Ricordiamo che un numero bicomplesso $x=x_{1} v_{1}+x_{2} v_{2}$ è nullo se ̀̀ $x_{1}=x_{2}=0$, è un divisore dello zero se è nulla una delle sue coordinate $x_{1}, x_{2}$. Se è $x_{1}=0, x$ si dirà divisore dello zero del $1^{\circ}$ sistema, se è $x_{2}=0, x$ si dirà, invece, divisore dello zero del $2^{\circ}$ sistema. I punti dello spazio complesso rappresentativo delle coppie di numeri bicomplessi $(x, y)$, con $x$ e $y$ divisori dello zero di nno stesso sistema o nulli sono, evidentemente $i$ punti del piano $p_{1}$ e del piano $\rho_{2}$. Per tal fatto $i$ piani paralleli a $\rho_{1}$ o a $\rho_{2}$ si diranno seminulli. Essi sono i piani propri passanti per $r^{\prime}$ o per $r^{\prime \prime}(\%)$

Le curve appartenenti ad un piano seminullo si diranno seminulle. In base a quanto abbiamo detto nel $\mathrm{n}$. precedente possiamo affermare che:

(7) Pertanto i piani che qui si chiamano seminulli coincidono con i piani che B. SEGRE ha chiamato mulli. Essi derivano dalla presenza dei divisori dello zero nell' algebra $C^{\prime}$ dei numeri bicomplessi. Ne segue che le linee che qui vongono chiamate seminulle sono le linee bicaratteristiche di B. SEGRE (Loc. eit. (1), $\mathrm{n}^{\circ}$ 6). 
II') Ogni superficie caratteristica dell' $\mathrm{S}_{+}$complesso contiene due sistemi di curve analitiche seminulle. Questa proprietr è caratteristica per dette superficie.

5. Un teorema dei fili analitiei. - Sia $f(t)$ una funzione olomorfa com. plessa della variabile reale $t$ data da

$$
f(t)=h(t)+-g(t) i
$$

con $h(t)$ e $g(t)$ funzioni reali olomorfe della variabile reale $t$, definite in un intervallo $\left(t^{\prime}, t^{\prime \prime}\right)$, e quindi sviluppabili in serie di potenze

$$
\begin{aligned}
& h(t)=\sum_{m}^{\mathrm{S}} a_{m}\left(t-t_{1}\right)^{m} \\
& g(t)=\sum_{m b}^{\mathrm{\Sigma}} b_{m}\left(t-t_{1}\right)^{m}
\end{aligned}
$$

nell' intorno di ogni punto $t_{1}$ di $\left(t^{\prime}, t^{\prime \prime}\right)$. In detto intorno per la $f(t)$ si ha lo sviluppo in serie

$$
f(t)=\sum_{w}\left(a_{m}+b_{m} i\right)\left(t-t_{1}\right)^{m}
$$

Posto $z=t+i q$, consideriamo la serie, che si ottiene dalla (9) sostituendo alla variabile reale $t$ la variabile complessa $z$,

$$
\sum_{n}\left(a_{m}+b_{m} i\right)\left(z-t_{i}\right)^{m}
$$

Questa serie, al variare di $t_{1}$ in $\left(t^{\prime}, t^{\prime \prime}\right)$, fornisce infiniti elementi di una funzione analitica della variabile complessa $z$ con i centri nei punti $t_{\text {: }}$ del segmento dell' asse reale $t$, posto nel piano di GaUss $(t, q)$, avente per estremi i punti $t^{\prime}$ e $t^{\prime \prime}$. I cerchi di convergenza di dette infinite serie costituiranno un campo $C$ a cui appartiene il segmento $t^{\prime} t^{\prime \prime}$, e in $C$ definiscono una funzione olomorfa (uniforme) $f(\approx)$ della variabile complessa $\approx$ che lung, detto segmento si riduce alla data funzione $f(t)$. La $f(z)$ dicesi il prolungamento della $f(t)$ nel corpo dei numeri complessi.

Ciò ricordato, consideriamo quattro funzioni complesse olomorfe, non tutte oostanti,

$$
x_{1}(t), \quad x_{2}(t), \quad y_{1}(t), \quad y_{2}(t)
$$

della variabile reale $t$, definite in uno stesso intervallo $\left(t^{\prime}, t^{\prime \prime}\right)$, e le funzioni loro prolungamento nel corpo complesso

$$
x_{1}(z), \quad x_{2}(z), \quad y_{1}(z), \quad y_{2}(z) .
$$

Indichiamo con $C$ un campo contenuto nei campi di esistenza delle funzioni (12) e contenente il segmento $t^{\prime} t^{\prime \prime}$. 
Mentre la variabile reale $t$ descrive l'intervallo $\left(t^{\prime}, t^{\prime \prime}\right)$, il punto $P$ dell' $S_{4}$ complesso di coordinate (11) descrive un insieme continuo di dimensione reale 1 , che dicesi un tratto di filo analitico dell' $S_{4}$ complesso. (Secondo una nomenclatura introdotta da CORRADO SEgRE, una varietà di uno spazio con. plesso di dimensioni reali $1,2,3$ dicesi filo, tela, trivarietà, rispettivamente).

Mentre la variabile complessa $z$ descrive il campo $C$, il punto di $S_{4}$ di coordinata (12) descrive un tratto di curva analitica (di dimensione complessa 1 e di dimensione reale 2). Ł̀ evidente che questo tratto di curva, diciamo $l_{1}$, contiene il tratto di filo $l$ sopra considerato, dunque:

III) Un tratto di filo analitico appartiene sempre ad un tratto di curva analitica determinato dal filo.

Questa proposizione è l'estensione ai fili analitici di una proprietà dei fili iperalgebrici dovuta a CoRRado SEgRe.

Cio posto, supponiamo che il tratto di filo $l$ sia tale che l'intorno di ogni suo punto $P$ non appartenga ad un piano seminullo, o, come diremo, che non sia seminullo. Anche il tratto di curva $l_{1}$ sarà di conseguenza non seminullo. Proiettando $l_{1}$ da $r^{\prime \prime}\left(r^{\prime}\right)$ sul piano $\rho_{1}\left(\rho_{2}\right)$ si ottiene un tratto di curva analitica $f_{1}\left(f_{2}\right)$. Precisamente $f_{1}\left(f_{2}\right)$ è rappresentata in $\rho_{1}\left(\rho_{2}\right)$ dalle prima e terza (seconda e quarta) delle equazioni (12). L'intersezione $P$ di due piani $P_{1} r^{\prime \prime}$ e $P_{2} r^{\prime}$, con $P_{1}\left(P_{2}\right)$ variabile in $f_{1}\left(f_{2}\right)$, deserive un pezzo di superficie caratteristica $F$ contenente il tratto di curva $l_{1}$ e quindi il tratto di filo $l$.

Supponiamo ora che $F_{1}$ sia una superficie caratteristica contenente il tratto di filo $l$ e quindi il tratto di curva $l_{1}$ determinato da $l$; le proiezioni di un pezzo di $F_{1}$ contenente $l_{1}$ da $r^{\prime \prime}$ e $r^{\prime}$ su $\rho_{1}$ e $\rho_{2}$ dovranno essere due tratti di curve contenenti $f_{1}$ e $f_{z}$ perchè $f_{1}$ e $f_{2}$ sono le proiezioni di $l_{1}$. Ma allora sarà $F=F_{1}$, perchè anche $F$ è proiettata da $r^{\prime \prime}$ e $r^{\prime}$ su $\rho_{1}$ e $\rho_{2}$ in $f_{1}$ e $f_{z}$. Dunque per un tratto di filo analitico passa un solo pezzo di superficie caratteristica.

Mettiamoci ora nell'ipotesi che il tratto di filo $l$ sia seminullo, e per fissare le idee supponiamo che appartenga ad un piano seminullo passante per $r^{\prime \prime}$, cioè del $2^{\circ}$ sistema. Proiettando $l$ da $r^{\prime \prime}$ su $\rho_{\text {s }}$ si ottiene un punto $P_{1}(a, 0, b, 0)$, il che equivale ad affermare che in questo caso le funzioni $x_{1}(t)$ e $y_{1}(t)$ sono due costanti $a$ e $b$. Si conduca per $P_{1}$, nel piano $\rho_{1}$, un qualunque tratto di curva analitica $f_{1}$. In corrispondenza ai due tratti di eurve analitiche $f_{1}$ e $f_{2}$, avremo una superficie caratteristica $F$, intersezione delle varietà che si ottengono proiettando $f_{1}$ e $f_{2}$ da $r^{\prime \prime}$ e $r^{\prime}$, passante per il tratto di filo $l$. Concludendo si ha il teorema:

IV) Per un tratto di filo analitico non seminullo passa una ed una sola superficie caratteristica dell' $\mathrm{S}_{4}$ complesso. 
Per un tratto di filo analitico seminullo passano infinite superficie caratteristiche dell' $\mathrm{S}_{4}$ complesso $\left({ }^{\circ}\right)$.

6. Le trasformazioni caratteristiche dell' $\boldsymbol{S}_{4}$ complesso. - Una trasfor. mazione bicomplessa su due variabili

$$
X=X(x, y), \quad Y=Y(x, y)
$$

si dirà caratteristica se le funzioni $X(x, y)$ e $Y(x, y)$ delle due variabili bicomplesse indipendenti $x$ e $y$ sono totalmente derivabili rispetto ad $x$ e rispetto ad $y$ e, applicate ad un campo convenientemente ristretto dell' $S_{4}$ complesso, risultano invertibili e tali da definire $y$ e $x$ come funzioni totalmente derivabili di $X$ e $Y$. Se dieiamo omologhi dne punti di $S_{4}$ immagini di due coppie $(x, y)$ e $(X, Y)$ legate dalle [3], resta determinata fra dne campi di $S_{4}$ una trasformazione biunivoca $w$ che si dirà una trasformazione caratteristica dell' $\mathrm{S}_{4}$ complesso.

Posto

$$
\left\{\begin{array}{l}
X=X_{1} v_{1}+X_{2} v_{2} \\
Y=Y_{1} v_{1}+Y_{2} v_{2}
\end{array}, \quad\left\{\begin{array}{l}
x=x_{1} v_{1}+x_{2} v_{2} \\
y=y_{1} v_{1}+y_{2} v_{2}
\end{array}\right.\right.
$$

le $X_{1}, Y_{1}$ saranno funzioni olomorfe delle sole variabili complesse indipendenti $x_{1}$ e $y_{1}$; inrece $X_{2}$ e $Y_{2}$ saranno funzioni olomorfe delle sole variabili indipendenti $x_{2}$ e $y_{2}$. Sicchè posto

$$
\left\{\begin{array} { l } 
{ X _ { 1 } = X _ { 1 } ( x _ { 1 } , y _ { 1 } ) } \\
{ Y _ { 1 } = Y _ { 1 } ( x _ { 1 } , y _ { 1 } ) }
\end{array} \quad \left\{\begin{array}{l}
X_{2}=X_{2}\left(x_{2}, y_{2}\right) \\
Y_{2}=Y_{2}\left(x_{2}, y_{2}\right)
\end{array},\right.\right.
$$

saranno le $\left[3^{\prime}\right]$ le equazioni della trasformazione caratteristica $w$ dell' $S_{4}$ com-

( ${ }^{8}$ B. Segre ha dimostrato nella sua memoria citata in $\left({ }^{1}\right), \mathrm{n}^{\circ} 5, \mathrm{p} .66$. che per m tratto regolare di linea analitica (reale) assegnata comunque in $\mathrm{S}_{4}$ passa uno ed un sol pezzo di superficie caratteristica. Nel seguente $\mathrm{n}^{\circ} 6$ il SEGRe ha osserrato che la proposizione enunciata vale anche nel campo complesso per una linea analitica assegnata non reale, purchè non sia bicaratteristica e che la superficie che si viene a determinare non sarà in generale reale (Nota (21) della pag. 68). Tenendo conto di quanto abbiamo osservato nella nota (T), si scorge che la prima parte del teorema IV) non è altro che la proposizione di B. SEGre, su ricordata, nel campo complesso. La eccezionalità delle linee bicaratteristiche, cioè dei fili seminulli, che nella dimostrazione del SEGRE proveniva dall'annullarsi identicamente di una certa espressione, nella nostra dimostrazione proviene dal fatto che un filo seminullo $\grave{e}$ prolettato da $r^{\prime \prime}$ e $r^{*}$, in uno deị piani coordinati $\rho_{1}$ e $\rho_{2}$, in un punto. Da quanto sopra si è osservato si conclude che le superficie non reali dell' $S_{4}$ complesso a cui il SEGRe accenna nella citata nota ${ }^{(21)}$ di pag. 68 , non sono altro che le superficie immagini delle funzionì di variabile bicomplessa olomorfe secondo Suorza Dragon o totalmente derivabili. 
plesso. Le $\left[3^{\prime}\right]$, separatamente, determinano nei piani coordinati $p_{1}\left(x_{1}, y_{1}\right)$ e $\rho_{2}\left(x_{2}, y_{2}\right)$ due trasformazioni $w_{1}$ e $w_{2}$ in cui si corrispondono le proiezioni $P_{1}$ e $P_{1}^{\prime}, P_{2}$ e $P_{2}^{\prime}$ di due punti omologhi $P$ e $P^{\prime}$ in $w$ fatte, rispettivamente, da $r^{\prime \prime}$ e $r^{\prime}$.

Un campo piano seminullo, cioè appartenente ad un piano seminullo del $1^{\circ}$ o del $2^{\circ}$ sistema, sarà trasformato da $w$ evidentemente in un campo piano seminullo dello stesso sistema, perchè punti del piano seminullo $r^{\prime \prime} P_{1}\left(r^{\prime} P_{2}\right)$ rengono trasformati da $w$ in punti del piano seminullo $r^{\prime \prime} P_{1}^{\prime}\left(r^{\prime} P_{2}^{\prime}\right)$. Viceversa se una trasformazione analitica invertibile dell' $S_{4}$ complesso deve trasformare campi seminulli in campi seminulli dello stesso sistema, subordinerà in convenienti insiemi di piani seminulli, cioè passanti per $r^{\prime} \circ r^{\prime \prime}$, una trasformazione analitica e quindi subordinerà una trasformazione analitica in ciascuno dei piani coordinati $\rho_{1}$ e $\rho_{2}$, chiamando omologhi in $\rho_{1}\left(\rho_{2}\right)$ due punti sezioni di piani seminulli omologhi passanti per $r^{\prime \prime}\left(r^{\prime}\right)$. Le equazioni di tali trasformazioni sono del tipo $\left[\mathfrak{3}^{\prime}\right]$ e quindi la trasformazione analitica assegnata è una trasformazione caratteristica. Si ha pertanto:

V) Condizione necessaria e sufficiente affinchè una trasformazione anali tica invertibile dell' $\mathrm{S}_{4}$ complesso sia una trasformazione caratteristica, è che trasformi campi seminulli in campi seminulli dello stesso sistema $\left({ }^{9}\right)$.

Ne segue che: una trasformazione caratteristica trasforma curve semimulle in curve seminulle dello stesso sistema, e, quindi, tenendo conto del teorema $I^{\prime}$ ) del n. 4 , si ricava che:

VI) Una trasformazione caratteristica dell' $\mathrm{S}_{4}$ complesso trasforma superficie caratteristiche in superficie caratteristiche, trasformando le curve semi. mulle dell'una nelle curve seminulle dell' altra appartenenti allo stesso sistema.

Si osservi che se una trasformazione caratteristica $w$ trasforma una superficie caratteristica $F$ in una superficie (caratteristica) $F^{\prime}$ e $f_{1}, f_{2}$ e $f_{1}^{\prime}, f_{2}^{\prime}$ sono le proiezioni di $F$ e $F^{\prime}$ su $\rho_{1}$ e $\rho_{2}$ da $r^{\prime \prime}$ e $r^{\prime}$, le $\left[3^{\prime}\right]$ a sinistra (a destra) devono trasformare $f_{1}\left(f_{2}\right)$ in $f_{1}^{\prime}\left(f_{0}^{\prime}\right)$. Se, in particolare, la $F$ è un pezzo di piano caratteristico, nel qual caso le $f_{1} \ominus f_{2}$ sono segmenti rettilinei, perchè $F^{\prime}$ sia pur un pezzo di piano (caratteristico) occorre e basta che auche $f_{i}^{\prime}$ e $f_{2}^{\prime}$ siano segmenti rettilinei. Ora perchè le trasformazioni, in $p_{i}$ e $p_{2}, w_{1}$ e $w_{2}$, di equazioni [3'], trasformino segmenti rettilinei in segmenti

(9) Questa proposizione generalizan la seguente proposizione di B. SEGRE: Le trasfor. mazioni pseudoconformi possono venire caratterizate fra le trasformazioni puntuali analitiche (e reali) dell' $\mathrm{S}_{4}$ in sè stesso dalla proprietì di trasformare piani nulli in piani nulli associando fro loro piani dello stesso sistema (Loc. cit. (1), p. 85). 
rettilinei, occorre e basta che $X_{1}, Y_{1}\left(X_{2}, Y_{2}\right)$ siano funzioni lineari fratte di $x_{1}, y_{1}\left(x_{2}, y_{2}\right)$. Ne segue immediatamente che:

$\left.\mathrm{VI}^{\prime}\right)$ Le trasformazioni caratteristiche dell' $\mathrm{S}_{4}$ complesso trasformanti regioni piane caratteristiche in regioni piane (caratteristiche) sono le immagini delle trasformazioni bicomplesse rappresentate da sostituzioni lineari fratte nelle ane variabili bicomplesse $\mathrm{x}$ e $\mathrm{y}\left({ }^{10}\right)$.

7. Superficie semicaratteristiche. - Una superficie o un pezzo di superficie dell' $S_{4}$ complesso si dirà semicaratteristica del $1^{\circ}\left(2^{\circ}\right)$ sistema se proiettata da $r^{\prime \prime}\left(r^{\prime}\right)$ su $\rho_{1}\left(\rho_{z}\right)$ dà, anzichè una regione a due dimensioni, un pezzo di curva che non si riduca ad un punto (nel qual onso la superficie è una regione piana seminulla).

Una superficie semicaratteristica del $1^{\circ}(20)$ sistema è secata da piani seminulli passanti per $r^{\prime \prime}\left(r^{\prime}\right)$ in curve seminulle, quindi ogni tale superficie o pezzo di superficie contiene un sistema di curve seminulle. E evidente che:

VII) Ina trasformazione caratteristica dell' $\mathrm{S}_{4}$ complesso trasforma una. superficie semicaratteristica del $1^{\circ}\left(2^{\circ}\right)$ sistena in una superficie semicaratteristica dello stesso sistema, trasformando le curve seminulle della prima nelle curve seminulle della seconda.

8. Teorema fondamentale. - Sia $G$ una regione superficiale analitica dell' $S_{4}$ complesso, cioè una superficie o un pezzo di superficie analitica, e diciamo $G_{1}$ e $G_{2}$ le proiezioni di $G$ da $r^{\prime \prime}$ e $r^{\prime}$ su $f_{1}$ e $\rho_{2}$ rispettivamente. Se $G_{1} \quad$ o $G_{2}$ si riduce ad un punto sarà $G$ una regione piana posta in un piano seminullo, cioè una regione piana seminulla. Se $G$ non è una regione piana seminulla nè $G_{1}$ nè $G_{2}$ potranno ridursi ad un punto. In questa ipotesi $G_{1}$ o $G_{2}$ si ridurrà ad un tratto di curva quando e solo quando la $G$ è semicaratteristica. Ne segue che se $G$ non è seminulla nè semicaratteristica, $G_{1}$ e $G_{2}$ saranno due regioni piane di $\rho_{1}$ e $\rho_{2}$ nessuna delle quali può ridursi ad un punto o ad un tratto di curva. In questa ipotesi il piano proiettante da $r^{\prime \prime}$, o $r^{\prime}$, un panto generico $P$ di $G$, secherc̀ $G$ in un numero finito di punti e quindi, considerando la regione superficiale $\theta$ convenientemente piccola, possiamo supporre che il piano proiettante da $r^{\prime \prime}$, o $r^{\prime}$, ogni punto $P$ di $G$ sechi $G$ nel solo punto $P$. In tal caso la corrispondenza che nasce fra $G$ e $G_{1} \circ G_{2}$, chiamando omologo di $P$ il punto $P_{1} \circ P_{2}$ sua proiezione

(10) Questa proposizione generalizza la proposizione di B. SfGra del $n^{\circ} 25$, p. 90 della memoria citata in (1). 
da $r^{\prime \prime}$ o $r^{\prime}$ su $\rho_{1}$ o $\rho_{2}$. è biunivoca senza eccezioni. Ciò posto vogliamo dimostrare il seguente teorema:

VIII) Se G e $G^{\prime}$ sono due regioni superficiali dell $S_{4}$ complesso, non seminulle nè semicaratteristiche, sufficientemente piccole, e W è una corrispondenza biunivoca analitica fissata ad arbitrio fra esse, esiste una ed una sola trasformatione caratteristica fra due intorni quadrimensionali di $\mathrm{G} \dot{e} \mathrm{G}$ ' che trasformi $\mathrm{G}$ in $\mathrm{G}^{\prime}$, subordinandovi la corrispondenza $\mathrm{W}$ assegnata (1').

Infatti siano $P_{1}, P_{1}^{\prime}, P_{2}, P_{2}^{\prime}$ le proiezioni su $P_{1}$ e $\rho_{2}$ da $r^{\prime \prime}$ e $r^{\prime}$ di due punti $P$ e $P^{\prime}$ di $G$ e $G^{\prime}$ omologhi in $W$. Mentre $P$ descrive $G$, e quindi $P^{\prime}$ descrive $G^{\prime}$, i punti $P_{1}$ e $P_{i}^{\prime}$ del piano $P_{1}$ descriveranno le proiezioni $G_{1}$ e $G_{1}^{\prime}$ di $G$ e $G^{\prime}$ su $\rho_{1}$, e, per le ipotesi fatte, la corrispondenza biunivoca $W$ esistente fra $G$ e $G^{\prime}$ si proiettern in una corrispondenza biunivoca $W_{1}$ fra $G_{1}$ e $G_{1}^{\prime}$ in cui si corrisponderanno le proiezioni $P_{1}$ e $P_{1}^{\prime}$. Analoga osservazione si può fare relativamente alle proiezioni $G_{2}$ e $G_{z}^{\prime} \mathrm{di} G$ e $G^{\prime}$, sul piano $P_{2}$, da $r^{\prime}$. Fra $G_{2}$ e $G_{2}{ }^{\prime}$ avremo una corrispondenza biunivoca $W_{2}$, proiezione di $W$, in eui si corrisponderanno $P_{z}$ e $P_{z}^{\prime}$. Ciò posto diciamo $D\left(D^{\prime}\right)$ la regione quadrimensionale dell' $\mathrm{S}_{4}$ complesso costituita da tutti i punti $P\left(P^{\prime}\right)$ le cui proiezioni da $r^{\prime \prime}$ e $r^{\prime}$ su $\rho_{1}$ e $\rho_{2}$ cadano rispettivamente in $G_{1}$ e $G_{2}$ (in $G_{1}^{\prime}$ e $G_{2}{ }^{\prime}$ ).

Sia $P$ un punto di $D$ e $P_{1}, P_{2}$ le sue proiezioni da $r^{\prime \prime}$ e $r^{\prime}$ su $\rho_{1} \in \rho_{2}$. Siano $P_{1}^{\prime}$ e $P_{2}^{\prime}$ i punti, di $G_{1}^{\prime}$ e $G_{2}^{\prime}$, omologhi di $P_{1}$ e $P_{2}$ in $W_{1}$ e $W_{2}$ rispettivamente.

I piani $r^{\prime \prime} P_{1}^{\prime}$ e $r^{\prime} P_{z}^{\prime}$ si segheranno in un punto $P^{\prime}$ di $D^{\prime}$ che diremo omologo di $P$ in una corrispondenza $w$. La $w$ risulta biunivoca e analitica perchè sono biunivoche e analitiche le corrispondenze $W_{1}$ e $W_{2}$ proiezioni della corrispondenza biunivoca analitica $W$ assegnata fra $G$ e $G^{\prime}$. Inoltre la $w$ trasforma evidentemente $G$ in $G^{\prime}$ subordinandovi la corrispondenza $W$. Si osservi infine che se $P$ descrive una sezione piana seminulla di $D$, fatta per es. col piano seminullo $P_{1} r^{\prime \prime}$, il punto omologo $P^{\prime}$ di $D^{\prime}$ descriverà la sezione piana seminulla di $D^{\prime}$ fatta col piano seminullo $P_{1}^{\prime} r^{\prime \prime}$, e quindi, per: il teorema V), la trasformazione analitica $w$ è una trasformazione caratteristica. Il teorema risulta pertanto dimostrato per la parte che riguarda l'esistenza della trasformazione caratteristica trasformante $G$ in $G^{\prime}$ subordinandovi la $W$. Dimostriamo ora che una tale trasformazione è unica. Infatti

(11) Questa proposizione generalizza il teorema di B. Segre del $\mathrm{n}^{\circ} 24$, pag. 86 della memolia citata in (1). Il Segre impone che le due superficie $G$ e $G^{\prime}$ (per il Segre $F$ e $G$ ) non siano caratteristiche. Nel campo complesso questa condizione non è sufficiente: bisogna invece in questo caso imporre cho non siano semicaratteristiche nè seminulle. 
una trasformazione caratteristica che goda delle proprietà indicate subordinerà negl'insiemi di piani per $r^{\prime \prime}\left(r^{\prime}\right)$ proiettanti $i$ punti di $G$ e $G^{\prime}$, la trasformazione analitica che si ottiene proiettando la $W_{1}\left(W_{2}\right)$ e quindi tale trasformazione coinciderà con la $w$.

9. Un teorema relativo alle $V_{3}$ analitiche. - Sia $V_{3}$ una varietà analitica di $S_{4}$ a 3 dimensioni. Se $P$ è un suo punto, il piano seminullo $r^{\prime \prime} P$ la secherà in una ourva analitica $s_{1} 0$, per posizioni particolari di $V_{3}$ rispetto alla retta $r^{\prime \prime}$, in una regione piana $\sigma_{1}$. In questo secondo caso diremo che la $V_{3}$ contiene una regione piana seminulla del $1^{\circ}$ sistema.

Secando la $V_{3}$ con il piano seminullo $r^{\prime} P$, si ottiene una curva ana* litica $s_{z} 0$, in casi particolari, una regione piana $\sigma_{z}$, che si dirà una regione piana seminulla del $2^{\circ}$ sistema.

E evidente che se si trasforma la $V_{3}$ con una trasformazione caratteristica $w$, la $V_{3}^{\prime}$ trasformata sarà priva di regioni piane seminulle se è tale Ia $V_{3}$, perchè ogni eventuale regione piana seminulla di $V_{3}$ sarò trasformata da $\mathrm{w}$ in una regione piana seminulla (dello stesso sistema), della $\mathrm{V}_{3}$.

Mettiamoci nell'ipotesi che la $V_{3}$ sia priva di regioni piane seminulle. In tal caso per ogni punto $P$ di $V_{3}$ passa una curva seminulla $s_{\text {, }}$ del $1^{\circ}$ sistema ed una curva seminulla $s_{2}$ del $2^{\circ}$ sistema. Queste due curve si otten. gono secando la $V_{3}$ con i piani seminulli $r^{\prime \prime} P$ e $r^{\prime} P$. La $V_{3}$ possiede due sistemi $\infty^{2}$ di curve seminulle ohe indicheremo con $\left(s_{1}\right)$ e $\left(s_{z}\right)$. Mentre $P$ descrive $V_{3}$ le sue proíezioni $P_{1}$ e $P_{2}$ da $r^{\prime \prime}$ e $r^{\prime}$, su $\rho_{1}$ e $\rho_{2}$, descriveranno due regioni $\sigma_{1}$ e $\sigma_{2}$, nessuna delle quali può ridursi ad una curva, perchè se per es. fosse $\sigma_{1}$ una curva, $i$ punti della $V_{3}$ dovrebbero essere distribuiti negli $\infty^{1}$ piani proiettanti $i$ punti di $\sigma_{3}$ da $r^{\prime \prime}$, e quindi, per essere la $V_{3}$ a tre dimensioni, in $\infty^{1}$ di tali piani dovrebbe ammettere una regione piana, contro l'ipotesi che la $V_{3}$ sia priva di regioni piane seminulle.

Sia $P_{1}$ un punto di $\sigma_{1}$. Il piano $P_{1} r^{\prime \prime}$ segherà la $V_{3}$ in una curva $s_{1}$ di $\left(s_{1}\right)$. Questa proiettata da $r^{\prime}$ su $\rho_{z}$ darà una curva $f_{2}$ di $\sigma_{z}$ che diremo omologa di $P_{1}$ in una trasformazione analitica $\lambda$ che porta un punto $P_{1}$ di $\sigma_{1}$ in una curva $f_{z}$ di $\sigma_{2}$. Viceversa è evidente che l'inversa di $\lambda$ porta un punto $P_{2}$ di $\sigma_{2}$ in una curva $f_{1}$ di $\sigma_{1}$, curva che si ottiene proiettando da $r^{\prime \prime}$ su $P_{1}$ la curva $s_{z}$ sezione di $V_{3}$ con il piano $P_{2} r^{\prime}$. Se $\mathrm{P}_{1}$ e $\mathrm{P}_{2}$ sono le proiezioni di uno stesso punto $\mathrm{P} d i \mathrm{~V}_{3}$, la curva $\mathrm{f}_{2}$ passa per $\mathrm{P}_{2}$ e la curva $\mathrm{f}_{1}$ passa per $\mathrm{P}_{1}$. 亡 evidente che la corrispondenza $\lambda$ e la $V_{3}$ si determinano a vicenda.

Ciò posto, consideriamo in $\sigma_{1}$ una curva analitica $g_{1}$. Mentre un punto $P_{1}$ descrive $g_{1}$, la curva seminulla $s_{1}$, sezione di $V_{3}$ con il piano $P_{1} r^{\prime \prime}$, descrive 
una superficie semicaratteristica $G$ di $V_{3}$, appartenente al $1^{0}$ sistema, perchè $\grave{e}$ proiettata da $r^{\prime \prime}$ su $p_{1}$ nella curva $g_{1}$ data. Sicchè per ogni curva $g_{k}$ di $\sigma_{1}$ si ha in corrispondenza una superficie semiearatteristica $G$ del $1^{\circ}$ sistema appartenente a $V_{3}$. (Analoga osservazione si fa relativamente alle curve $g_{2}$ di $\sigma_{2}$ alle quali corrisponderanno superficie semicaratteristiche del $2^{\circ}$ sistema appartenenti alla $V_{3}$ ). La superficie semicaratteristica $G$, di cui sopra, per essere caratteristica occorre e basta che sia semicaratteristica anche del $2^{\circ}$ sistema, cioè che proiettata da $r^{\prime}$ su $\rho_{2}$ dia una curva $g_{2}$. In questo caso le $\infty$ curve seminulle $s_{1}$ giacenti nei piani $P_{1} r^{\prime \prime}$, con $P_{1}$ in $g_{1}$, vengono tutte proiettate da $r^{\prime}$ su $\rho_{1}$ nella curva $g_{2}$. Sicchè $g_{2}$ è la curva omologa in $\lambda$ di ogni punto $P_{1}$ di $g_{1}$. La $G$ conterrà inoltre $\infty^{1}$ curve $s_{z}$, quelle poste nei piani $P_{2} r^{\prime}$, con $P_{2}$ in $g_{2}$, e tutte queste curve saranno proiettate da $r^{\prime}$ su $\rho_{1}$ nella curva $g_{1}$. Quindi ad ogni punto $P_{z}$ di $g_{2}$ corrisponde, per l'inversa di $\lambda$, la curva $g_{1}$. Viceversa è evidente che se si hanno in $\sigma_{1}$ e $\sigma_{2}$ due curve analitiche $g_{1}$ e $g_{2}$ tali che ad ogni punto di $g_{1}\left(g_{2}\right)$ corrisponde per la $\lambda\left(\lambda^{-1}\right)$ la curva $g_{z}\left(g_{1}\right)$, la superficie caratteristica riempita dai punti $P$ aventi le proiezioni $P_{1}\left(P_{2}\right)$ [da $r^{\prime \prime}\left(r^{\prime}\right)$ su $\left.\rho_{1}\left(g_{2}\right)\right]$ appartenenti a $g_{1}\left(g_{2}\right)$, appartiene alla $V_{3}$. Dunque:

IX) Condizione necessaria e sufficiente affinchè una $\mathrm{V}_{3}$ analitica contenga una superficie caratteristica è che la corrispondente trasformazione $\lambda$ ammetta due curve singolari $\mathrm{g}_{1}$ e $\mathrm{g}_{2}$, cioè tali che ad ogni punto di $\mathrm{g}_{1}\left(\mathrm{~g}_{2}\right)$ corrisponda $\mathrm{g}_{2}\left(\mathrm{~g}_{1}\right)$.

10. Ipersuperficie analitiche caratteristiche. - Una ipersuperficie analitica $V_{2}$ priva di regioni piane seminulle, si dirà caratteristica se è costituita da un sistema $\infty^{1}$ di superficie caratteristiche. Se la $V_{3}$ considerata nel n. 8 è caratteristica, la regione $\sigma_{1}\left(\sigma_{2}\right)$ dovrà contenere un sistema $\infty^{1}$ di curve analitiche $g_{1}\left(g_{2}\right)$ tali che la corrispondenza $\lambda\left(\lambda^{-1}\right)$ deve far corrispondere ad ogni punto $P_{1}\left(P_{2}\right)$ di una assegnata curva $g_{1}\left(g_{2}\right)$ del sistema, nna determinata curva $g_{2}\left(g_{1}\right)$, sicchè fra $\mathrm{i}$ due sistemi $\infty^{1}$ di curve $g_{1}$ e $g_{2}$ la trasformazione $\lambda$ determina una corrispondenza biunivoca $L$. Alle varie coppie di curve $\left(g_{1}, g_{2}\right)$ corrispondentisi in $L$ (coppie di curve singolari per la $\lambda$ ) corrispondono le superficie caratteristiche contenute nella $V_{3}$ caratteristica considerata, nel senso precisato nel n. 8 .

Sia $V_{3}^{\prime}$ un'altra ipersuperfieie caratteristica di $S_{4}$ e siano $\sigma_{1}^{\prime}, \sigma_{2}^{\prime}, g_{1}^{\prime}, g_{2}^{\prime}$, $\lambda^{\prime}$ e $L^{\prime}$ le regioni di $\rho_{1}$ e $\rho_{2}$, le curve di $\sigma_{2}$ e $\sigma_{2}$ e le trasformazioni che per $V_{3}^{\prime}$ hanno lo stesso significato che per la $V_{3}$ hanno $\sigma_{1}, \sigma_{z}, g_{1}, g_{2}, \lambda \in L$.

Sia $w_{1}\left(w_{2}\right)$ una trasformazione analitica invertibile nel piano $\rho_{1}\left(\rho_{2}\right)$ che trasformi $\sigma_{1}\left(\sigma_{2}\right)$ in $\sigma_{1}{ }^{\prime}\left(\sigma_{2}{ }^{\prime}\right)$ e tale che trasformi il sistema di curve anali- 
tiche $g_{1}$ di $\sigma_{1} \quad \mid g_{2}$ di $\sigma_{2} \mid$ nel sistema di curve $g_{1}^{\prime}$ di $\sigma_{1}^{\prime}$ (di curve $g_{2}^{\prime}$ di $\sigma_{2}{ }^{\prime}$, portando coppie di curve omologhe in $L$ in coppie di curve omologhe in $L^{\prime}$.

Se diciamo $D$ il canpo quadridimensionale formato dai punti $P$ con le proiezioni $P_{1}$ e $P_{z}$ da $r^{\prime \prime}$ e $r^{\prime}$ su $\rho_{1}$ e $P_{2}$ appartenenti rispettivamente a $\sigma_{1}$ e $\sigma_{z}$; le trasformazioni analitiche $w_{1}$ e $w_{z}$ determinano una trasformazione caratteristica $w$ che opera su $D$ : un punto $P$ di $D$ è trasformato da $w$ nel punto intersezione dei piani $r^{\prime \prime} P_{1}^{\prime}, r^{\prime} P_{2}^{\prime}$, essendo $P_{1}^{\prime}$ e $P_{\mathrm{o}}^{\prime}$ i trasformati di $P_{1}$ e $P_{2}$ in $w_{1}$ e $w_{2}$. Se $P$ appartiene a $V_{2}, P_{1}$ e $P_{2}$ saranno due punti appartenenti a due curve $g_{1}$ e $g_{2}$ omologhe in $L$. Ne segue che i loro trasformati mediante $w_{1}$ e $w_{2}$ saranno due punti $P_{1}^{\prime}$ e $P_{z}^{\prime}$ appartenenti a due curve $g_{1}{ }^{\prime}$ e $g_{2}{ }^{\prime}$ omologhe in $L$, e quindi il punto $P^{\prime}$ apparterrà a $V_{3}^{\prime}$. La $w$ trasforma, per* tanto, la $V_{3}$ nella $V_{3}$. Dunque:

$\mathrm{X})$ Date due ipersuperficie caralteristiche, è sempre possibile trasformarle una nell' altra mediante una trasformazione caratteristica.

NотA. - Si osservi che esistono infinite trasformazioni caratteristiche che portano la $V_{3}$ nella $V_{3}^{\prime}$, perchè esistono infinite trasformazioni $w_{1}$ e $w_{2}$ soddisfacenti alle condizioni poste nella dimostrazione del toorema X). Infatti per determinare due tali corrispondenze $w_{1}$ e $w_{2}$ si può procedere nel seguente modo: Si fissino, in primo luogo, due superficie analitiche $H$ e $H^{\prime}$ non semicaratteristiche nè seminulle contenute rispettivamente in $V_{3}$ e $V_{3}^{\prime}$. Se $P$ è un punto di $H$, e quindi di $V_{3}$, le proiezioni $P_{1}$ e $P_{2}$ su $\sigma_{1}$ e $\sigma_{2}$ apparterranno a due curve $g_{1}$ e $g_{2}$ omologhe in $L$, sicchè chiamando omologhi due punti $P_{2}$ e $P_{2}$ proiezioni su $\rho_{1}$ e $\rho_{2}$ da $r^{\prime \prime}$ e $r^{\prime}$ di uno stesso punto $P$ di $H$, resta determinata una trasformazione analitica $h$ fra $\sigma_{1}$ e $\sigma_{2}$ che trasforma le curve $g_{1}$ nelle omologhe $g_{2}$ per la trasformazione $L$. Mentre $P_{1}$ descrive una curva $g_{1}$, e quindi l'omologo $P_{2}$ in $h$ descrive la curva $g_{2}$ omologa di $g_{4}$ in $L$, il punto $P$ di $I I$ descriverà una curva $g$ appartenente alla superficie caratteristica $G$ corrispondente alla coppia di curve $g_{1}$ e $g_{2}$. Analoghe osservazioni si facciano relativamente alla superficie $H^{\prime}$ di $V_{3}^{\prime}$, usando gli stessi simboli con gli apici.

Ciò posto, fra le superficie $H$ ed $H^{\prime}$ fissiamo una corrispondenza analitica biunivoca $W$ che trasformi il sistema di curve $g$ di $H$ nel sistema di curve $g^{\prime}$ di $H^{\prime}$.

Proiettando da $r^{\prime \prime}$ su $\rho_{1}\left(\right.$ da $r^{\prime}$ su $\left.\rho_{2}\right)$ le coppie $\left(P, P^{\prime}\right)$ omologhe in $W$ si ottengono coppie di punti $\left(P_{1}, P_{1}^{\prime}\right),\left(P_{2}, P_{2}^{\prime}\right)$ omologhe in una corrispondenza analitica $w_{1}\left(w_{2}\right)$ invertibile. Queste due trasformazioni soddisfano alle con. dizioni di cui sopra.

Si osservi, anzi, che la trasformazione caratteristica $w$ determinata da $w_{1}$ 
e $w_{2}$ è la trasformazione che trasforma la $H$ nella $H^{\prime}$ subordinandovi la $W$. Questa stessa trasformazione trasforma la $V_{3}$ nella $V_{3}^{\prime}$.

11. Gli $S_{3}$ caratteristici. - Sia $S_{3}$ un iperpiano dell' $S_{4}$ complesso $\sigma$, distinto dall' $S_{3}$ improprio. Se $S_{3}$ passa per $r^{\prime}$ o $r^{\prime \prime}$, conterrà un fascio di piani seminulli. Un tale $S_{3}$ non è pertanto caratteristico, perchè, per definizione, una $V_{3}$ carattexistica non deve contenere regioni piane seminulle.

Se $S_{3}$ non passa nè per $r^{\prime}$ nè per $r^{\prime \prime}$, secherà queste due rette in due punti $R^{\prime}$ e $R^{\prime \prime}$. Tutti i piani di $S_{4}$ passanti per $R^{\prime}$ e $R^{\prime \prime}$ sono piani caratteristici e di questi un fascio appartiene all' $S_{3}$ considerato. Un tale $S_{3}$ è, pertanto, una varietà $V_{3}$ caratteristica perchè contiene $\infty^{1}$ superficie caratteristiche. Dunque:

XI) Gli $\mathrm{S}_{3}$ caratteristici dell' $\mathrm{S}_{4}$ complesso sono tutti e soli gli $\mathrm{S}_{3}$ non passanti nè per $\mathrm{r}^{\prime}$ nè per $\mathrm{r}^{\prime \prime}$.

Dal teorema $X$ ) segue che:

XII) Ogni $V_{a}$ caratteristica è trasformabile con una trasformazione caratteristica in un pezzo di iperpiano secante $\mathrm{r}^{\prime}$ ed $\mathrm{r}^{\prime \prime}$ in un punto $\left(^{12}\right)$.

\section{\& II.}

1. Rappresentazione reale dei numeri bicomplessi. - Rappresentiamo la variabile bicomplessa $x=x_{1} v_{1}+x_{2} v_{2}=\left(x_{1}{ }^{\prime}+x_{1}{ }^{\prime \prime} i\right) v_{1}+\left(x_{2}{ }^{\prime}+x_{2}{ }^{\prime \prime} i\right) v_{2}$ nel punto $X\left(x_{1}{ }^{\prime}, x_{1}^{\prime \prime}, x_{2}{ }^{\prime}, x_{2}{ }^{\prime \prime}\right)$ di un $S_{4}$ reale euclideo. Questo punto si può considerare pure come l'immagine del punto $\left(x_{1}, x_{2}\right)$ del piano coordinato $\sigma_{1}\left(x_{1}, x_{2}\right)$ dell' $S_{4}$ complesso considerato nel $\S \mathrm{I}$, per rappresentare le coppie di variabili bicomplesse $(x, y)$. Analogamente rappresentiamo la variabile bicomplessa $y=y_{1} v_{1}+y_{2} v_{2}=\left(y_{1}^{\prime}+i y_{1}^{\prime \prime}\right) v_{1}+\left(y_{2}^{\prime}+i y_{2}^{\prime \prime}\right) v_{2}$ nel punto $\boldsymbol{Y}\left(y_{1}^{\prime}, y_{1}^{\prime \prime}, y_{2}^{\prime}, y_{2}^{\prime \prime}\right)$ dello stesso $S_{4}$ reale eqclideo. Questo punto si può considerare pure l'im. magine del punto $\left(y_{1}, y_{2}\right)$ del piano coordinato $\sigma_{2}\left(y_{1}, y_{2}\right)$ dell' $S_{4}$ complesso.

Ciò posto, osserviamo che assegnare nell' $S_{4}$ complesso un punto $P$, immagine di una coppia $(x, y)$ di numeri bicomplessi, equivale ad assegnare due punti $X$ e $Y$ nell' $S_{4}$ reale, immagini dei due numeri bicomplessi $x$ e $y$.

Consideriamo nell' $S_{4}$ complesso il tratto di filo analitico di equazioni

$$
x_{1}=x_{1}(t), \quad x_{2}=x_{2}(t), \quad y_{1}=y_{1}(t), \quad y_{2}=y_{2}(t)
$$

(12) Per questa proprieta le $V_{3}$ caratteristiche si diranno pure iperplanoidi dell' $S_{4}$ complesso. Esse generalizzano gl'iperplanoidi dell' $S_{4}^{\prime}$ reale. 
essendo $x_{1}, x_{2}, y_{1}, y_{2}$ funzioni complesse olomorfe della variabile reale $t$, definite in un intervallo $\left(t^{\prime}, t^{\prime \prime}\right)$, non tutte e quattro costanti. In corrispondenza ad ogni punto $P$ del tratto di filo considerato, diciamolo $l$, fissiamo nell' $S_{*}$ reale i dne punti $X$ e $Y$ di cui sopra. Mentre $P$ descrive $l$, i punti $X$ e $Y$ descriveranno due linee (reali) $l_{x}$ e $l_{y}$. Può, in particolare, $l_{x}\left(l_{y}\right)$ ridursi ad un punto: ciò avviene quando e solo quando le funzioni $x_{1}, x_{2}\left(y_{1}, y_{2}\right)$ sono entrambe costanti. Non possono però $l_{x}$ ed $l_{y}$ ridursi contemporaneamente ad un punto, perchè le funzioni (1) non sono, per ipotesi, tutte e quattro costanti. Per fissare le idee, supponiamo che $l_{x}$ sia effettivamente un tratto di linea. In questa ipotesi l'assegnare il filo l nell' $\mathrm{S}_{4}$. complesso equivale ad assegnare la linea 1 nell' $\mathrm{S}_{4}$ reale e una funzione bicomplessa $\mathrm{y}^{*}(\mathrm{x})$ lungo questo tratto di linea: quella che nel punto di $l_{x}$, rispondente ad un assegnato valore del parametro reale $t$, assume il valore bicomplesso $y_{1}(t) v_{1}+y_{2}(t) v_{2}$.

Nel caso che anche $l_{y}$ non si riduca ad un punto, chiamando omologhi due punti di $l_{x}$ e $l_{y}$ se corrispondono ad uno stesso valore del parametro reale $t$, si viene a stabilire fra $l_{x}$ e $l_{y}$ una corrispondenza analitica invertibile, e quindi, in questa ipotesi: l'assegnare il tratto di filo 1 nell' $\mathrm{S}_{4}$ complesso equivale ad assegnare due tratti di linee analitiche (regolari, aperte) dell' $\mathrm{S}_{4}$ reale e una corrispondenza analitica invertibile fra detti tratti.

$\mathrm{Si}$ osservi ora che l'assegnare nell' $S_{4}$ reale una regione superficiale (a due dimensioni complesse e a quattro dimensioni reali) equivale ad assegnare nell' $S_{4}$ reale una regione quadridimensionale ed una funzione $y(x)$ bicomplessa del punto variabile in detta regione; e se la regione superficiale assegnata nell' $S_{4}$ complesso è caratleristica, la funzione bicomplessa del punto variabile nella regione quadridimensionale dell' $S_{4}$ reale è totalmente deri. vabile o olomorfa secondo SconzA DRAGoNr.

Se la regione superficiale considerata contiene il tratto di filo $l$ la funzione bicomplessa $y(x)$ assumerà lungo la linea $l_{x}$ [che apparterrà alla regione quadridimensionale dell' $S_{4}$ reale dove $y(x)$ è definita] i valori della funzione bicomplessa $y^{*}(x)$ determinata lungo $l_{x}$ dello stesso filo $l$, per quanto sopra è stato osservato.

Le osservazioni fatte bastano per poter affermare che la prima parte del teorema IV) equivale alla risoluzione del problema dell' equivalenza di due tratti di linea reali dell' $\mathrm{S}_{4}$ reale, questione trattata e risolta nel modo più completo da G. Sconza Dragons nel $\$ 7$ della sua memoria citata in $\left({ }^{4}\right)$, (ovvero al teorema conclusivo del n. 40 di p. 635). Si osservi che le curve singolari a cui accenna lo Sconza nel n. 41 corrispondono ai fli seminulli. 
2. Equivalenza di due superficie dell' $S_{4}$ reale. - Per interpretare nell' $S_{4}$ complesso il problema dell'equivalenza di due superficie reali dell' $S_{4}$ reale, risolto dallo Sconza Dragon nel $\$ 8$ della sua memoria citata in $\left({ }^{+}\right)$, ossepviamo quanto segue.

Siano date quattro funzioni complesse olomorfe

$$
x_{1}=x_{1}(t, p), \quad x_{2}=x_{2}(t, p), \quad y_{1}=y_{1}(t, p), \quad y_{z}=y_{2}(t, p)
$$

delle due variabili reali indipendenti $t, p$ definite in un rettangolo $R$

$$
t^{\prime} \leqq t \leqq t^{\prime \prime}, \quad p^{\prime} \leqq p \leqq p^{\prime \prime}
$$

Mentre il punto $(t, p)$ descrive il rettangolo $R$. il punto $P\left(x_{1}, x_{2}, y_{1}, y_{2}\right)$ dell' $S_{4}$ complesso descriva ciò che si dice una tela analitica $\mathrm{T}$, cioè una varietà complessa dell' $S_{4}$ complesso a due dimensioni reali. Ciò equivale a supporre che le funzioni (2) non sono tutte e quattro costanti, nè si possono ridurre a quattro funzioni olomorfe di un solo parametro reale, nel qual caso il punto $P$ non descriverebbe una tela, ma un filo.

Mentre il punto $P$ descrive la tela $T$ i punti $X$ e $Y$ dell' $S_{4}$ reale, considerati nel n. precedente, descrivono due varietà analitiche reali $T_{x}$ e $T_{y}$ di dimensioni $\leqq 2$ (dimensioni reali).

La $T_{x}\left(T_{y}\right)$ si riduce ad un punto quando e solo quando le prime (le ultime) funzioni (2) sono costanti. Questi due casi non si possono verificare contemporaneamente, anzi se $T_{x}\left(T_{y}\right)$ si riduce ad un punto, $T_{y}\left(T_{x}\right)$ deve essere a due dimensioni, altrimenti la $T$ non potrebbe essere a due dimensioni.

La $T_{x}\left(T_{y}\right)$ si riduce ad una linea quando e solo quando le prime (le ultime) due funzioni (2) si riducono a due funzioni olomorfe di un sol para. metro reale. Può accadere che $T_{x}$ e $T_{y}$ siano entrambe linee. In questo caso le (2) sono del tipo

$$
x_{1}=x_{1}\left(s_{1}\right), \quad x_{2}=x_{2}\left(s_{1}\right), \quad y_{1}=y_{1}\left(s_{2}\right), \quad y_{2}=y_{2}\left(s_{2}\right)
$$

con $s_{1}$ e $s_{2}$ parametri reali, e la tela $T$ è proiettata dalla retta impropria del piano coordinato $\sigma_{2}\left(y_{1}, y_{2}\right)\left[\sigma_{1}\left(x_{1}, x_{2}\right)\right]$ sul piano $\sigma_{1},\left(\sigma_{2}\right)$ in un filo e preciśamente in quello che è rappresentato dalle prime (ultime) equazioni $\left(Z^{\prime}\right)$. La $T$ viene ad essere l'immagine complessa delle coppie di punti dei due fili $T_{x}$ e $T_{y}$ e rappresenta pertanto la funzione bicomplessa che assume in ogni punto di $T_{x}$ gl' infiniti valori bicomplessi rappresentati dai punti di $T_{y}$. Questo caso pertanto non offre alcun interesse.

Supporremo dunque che una almeno delle varietà $T_{x}$ e $T_{y}$ sia a due dimensioni, cioè una regione superficiale dell' $S_{4}$ reale. Per fissare le idee 
supporremo che sia di dimensione 2 la $T_{\infty}$, cioè supporremo che le prime dne funzioni (2) non si riducano a funzioni di un solo parametro reale. In quest'ipotesi l'assegnare la tela $\mathrm{T}$ dell' $\mathrm{S}_{4}$ complesso equivale ad assegnare nell' $\mathrm{S}_{4}$ reale una superficie reale $\mathrm{T}_{\mathrm{x}}$ e una funzione bicomplessa $\mathrm{y}^{*}(\mathrm{x})$ del punto variabile su $\mathrm{T}_{\mathrm{x}}$. Nel caso poi che anche la $T_{y}$ sia a 2 dimensioni, l'assegnare nell' $S_{4}$ complesso la tela $T$ equivale ad assegnare nell' $S_{4}$ reale due superficie $T_{x}$ e $T_{y}$ e una corrispondenza analitica invertibile fra di esse (chia* mando omologhi due punti rispondenti agli stessi valori dei parametri $t$ e $p$ ).

$\mathrm{Ne}$ segue che l'esistenza di una funzione $\mathrm{y}(\mathrm{x})$ bicomplessa olomorfa secondo SCORZA Dragont, definita in un intorno quadridimensionale di $\mathrm{T}_{\mathrm{x}}$, che su $\mathrm{T}_{\mathrm{x}}$ si riduca alla funzione $\mathrm{y}^{*}(\mathrm{x})$, o, nell'altra ipotesi, l'equivalenza delle superficie $\mathrm{T}_{\mathrm{x}}$ e $\mathrm{T}_{\mathrm{y}}$ dell' $\mathrm{S}_{4}$ reale, equivale, nell' $\mathrm{S}_{4}$ complesso all' esistenza di una superficie caratteristica passante per la tela $\mathrm{T}$.

Ora la tela $T$ può essere curvilinea o superficiale secondo che le funzioni (2), prolungate nel corpo complesso, danno le funzioni olomorfe

$$
x_{1}=x_{1}(z, q), \quad x_{2}=x_{2}(z, q), \quad y_{1}=y_{1}(z ; q), \quad y_{2}=y_{2}(z, q),
$$

delle variabili indipendenti $z$ e $q$, che si riducano o no a quattro funzioni olomorfe di una sola variabile complessa, cioè secondo che le $\left(2^{\prime \prime}\right)$ rappresentino nell' $S_{4}$ complesso un tratto di curva $f_{T}$ o una regione superficiale $F_{T}$. Nel primo caso la $T$ essendo a due dimensioni reali, come la $f_{T}$, coinciderà con questo tratto di curva, cioè la tela $\mathrm{T}$ è un tratto di curva. Nel secondo caso la tela $T$ non è un tratto di curva, ma appartiene ad una superficie $F_{T}$ da essa determinata.

Nel primo caso:

a) se il tratto di curva $f_{T}=T$ è seminullo, per esso passano infinite superficie caratteristiche.

b) se il tratto di curva $f_{T}=T$ non è seminullo, per esso passa nna sola superficie caratteristica.

Nel secondo caso:

c) se la superficie $F_{T}$ determinata dalla tela $T$ è caratteristica, per $T$ passa una ed una sola superficie caratteristica: la $F_{T}$.

d) se la $F_{T}$ non è caratteristica, per la tela $T$ non passa alcuna su. perficie caratteristica.

$\mathrm{Si}$ ricordi che la condizione necessaria e sufficiente perchè la superficie $F_{T}$ dell' $S_{4}$ complesso sia caratteristica è che la sua proiezione da $r^{\prime \prime}\left(r^{\prime}\right)$ su $\rho_{1}\left(\rho_{z}\right)$ sia una curva. Si osservi, pertanto, che nei casi b) e c) la proieqione della tela $T$ sn $\rho_{1}\left(\rho_{2}\right)$ da $r^{\prime \prime}\left(r^{\prime}\right)$ è curvilinea, cioè appartiene ad una curva. Concludendo si ha il teorema: 
I) Sia $\mathrm{T}_{\mathrm{x}}$ una superficie analitica reale dell' $\mathrm{S}_{4}$ reale $e \mathrm{y}^{*}(\mathrm{x})$ una funzione bicomplessa del punto variabile su $\mathrm{T}_{\mathrm{x}}$, e $\mathrm{T}$ la tela dell' $\mathrm{S}_{4}$ complesso deter. minata da $\mathrm{T}_{\mathrm{x}}$ e dalla funzione $\mathrm{y}^{*}(\mathrm{x})$. Il problema della determinazione di una funzione bicomplessa totalmente derivabile, o olomorfa secondo SCORzA DRAGONI, definita in un intorno quadridimensionale di $\mathrm{T}_{\mathrm{x}}$ che su $\mathrm{T}_{\mathrm{x}}$ si riduca alla $\mathrm{y}^{*}(\mathrm{x})$,

1) ammette infinite soluzioni se $\mathrm{T}$ è un tratto di curva seminulla;

2) amnette una e una sola soluzione se le proiezioni di $\mathrm{T} d a \mathrm{r}^{\prime \prime}$ e $\mathrm{r}^{\prime}$ su $\rho_{1}$ e $\rho_{2}$ appartengono ad un tratto di curva, senza ridursi ad un punto;

3) non ammette alcuna soluzione se T non presenta uno dei casi 1) e 2). Sotto altro aspetto il teorema I) si può enuneiare nel seguente modo. Siano

$$
x_{1}^{\prime}=x_{1}^{\prime}(t, p), \quad x_{1}^{\prime \prime}=x_{1}^{\prime \prime}(t, p), \quad x_{2}^{\prime}=x_{2}^{\prime}(t, p), \quad x_{2}^{\prime \prime}=x_{2}^{\prime \prime}(t, p)
$$

quattro funzioni olomorfe reali delle due variabili reali $t, p$, rappresentanti una superficie $T_{x}$ dell' $S_{4}$ reale, e

$$
y^{*}(x)=y_{1}(t, p) v_{1}+y_{2}(t, p) v_{2}
$$

una funzione bicomplessa del punto variabile su $T_{x}$ con $y_{1}(t, p)$ e $y_{2}(t, p)$ funzioni complesse olomorfe delle due variabili reali $t$ e $p$ definite, insieme alle $\left(T_{x}\right)$, in uno stesso rettangolo del piano reale $(t, p)$.

Siano

$$
x_{1}=x_{1}(z, q), \quad x_{2}=x_{2}(z, q)
$$

le funzioni che si ottengono prolungando nel corpo complesso le funzioni

$$
x_{1}=x_{1}^{\prime}(t, p)+x_{1}^{\prime \prime}(t, p) i, \quad x_{2}=x_{2}{ }^{\prime}(t, p)+x_{2}^{\prime \prime}(t, p) i
$$

e siano $y_{1}=y_{1}(z, q), y_{2}=y_{2}(z, q)$ le funzioni che si ottengono prolungando nel corpo complesso le funzioni $y_{1}=y_{1}(t, p), y_{2}=y_{2}(t, p)$. Ciò posto:

I') Condizione necessaria e "sufficiente affinchè esista una funzione bicom. plessa totalmente derivabile, o olomorfa secondo SCORZA DragonI, definita in un intorno quadridinensionale di $\mathrm{T}_{\mathrm{x}}$, che su $\mathrm{T}_{\mathrm{x}}$ si riduca alla funzione (3), $\dot{e}$ che le funzioni $\mathrm{x}_{1}$ e $\mathrm{y}_{1}\left(\mathrm{x}_{2}\right.$ e $\left.\mathrm{y}_{2}\right)$ delle due variabili complesse $\mathrm{z} e q$ si ri. ducano a due funzioni di una sola variabile complessa $\mathbf{z}_{1}\left(\mathbf{z}_{2}\right)$. Nel caso particolare che $\mathrm{x}_{1}$ e $\mathrm{y}_{1}\left(\begin{array}{llll}( & \mathrm{x}_{2} & e & \mathrm{y}_{2}\end{array}\right)$ siano costanti, esistono infinite funzioni bicomplesse soddisfacenti alle condizioni volute; se nessuna di queste due circostanze si verifica, esiste una sola funzione bicomplessa che soddisfa alle condizioni imposte. 
3. Equivalenza di due $V_{3}$ dell' $s_{4}$ reale. - Il problema dell' equivalenza di due $V_{3}$ reali dell' $S_{4}$ reale risolto dallo Scorza Dragoni nel $\$ 9$ della sua memoria citata in (1), interpretato nell' $S_{4}$ complesso, dà luogo a considerazioni del tutto analoghe a quelle svolte nel $\mathrm{n}$. precedente relativamente al problema dell' equivalenza di due superficie.

L'assegnare nell' $S_{4}$ reale una varietà reale tridimensionale $V_{\infty}$ rappresentata dalle quattro funzioni reali

$\left(V_{x}\right) \quad x_{1}^{\prime}=x_{1}^{\prime}(r, t, p), \quad x_{1}^{\prime \prime}=x_{1}^{\prime \prime}(r, t, p), \quad x_{3}^{\prime}=x_{2}^{\prime}(r, t, p), \quad x_{2}^{\prime \prime}=x_{2}^{\prime \prime}(r, t, p)$

olomorfe, delle variabili reali $r, t, p$, in un parallelopipedo dell' $S_{3}$ reale $(r, t, p)$, e una tunzione bicomplessa del punto variabile su $V_{\infty}$, che nel punto rispondente ai valori $r, t, p$ assume il valore bicomplesso

$$
y^{*}(x)=y_{1}(r, t, p) v_{1}+y_{2}(r, t, p) v_{2}
$$

essendo $y_{1}(r, t, p)$ e $y_{2}(r, t, p)$ due funzioni complesse olomorfe definite nel detto parallelopipedo, equivale ad assegnare nell' $S_{4}$ complesso la trivarietà analitica $V$ di equazioni

$$
\left\{\begin{array}{l}
x_{1}=x_{1}{ }^{\prime}(r, t, p)+x_{1}^{\prime \prime}(r, t, p) i \\
y_{1}=y_{1}(r, t, p)
\end{array},\left\{\begin{array}{l}
x_{2}=x_{2}{ }^{\prime}(r, t, p)+x_{2}{ }^{\prime \prime}(r, t, p) i \\
y_{2}=y_{2}(r, t, p)
\end{array}\right.\right.
$$

Inoltre l'esistenza di ana funzione bicomplessa $\mathrm{y}(\mathrm{x})$ totalmente derivabile, o olomorfa secondo SCORzA DRAGONI, definita in un intorno quadridimensionale di $\nabla_{\mathrm{x}}$ e che su $\mathrm{V}_{\mathrm{x}}$ si riduca alla funzione bicomplessa (6), equivale atl'esistenza di una superficie caratteristica dell' $\mathrm{S}_{4}$ complesso passante per la trivarietà $\mathrm{V}$.

Supponiamo che per la trivarietà $V$ passi una superficie caratteristica $F$, e siano $f_{\mathrm{t}}$ e $f_{2}$ le proiezioni di $F$ da $r^{\prime \prime}$ e $r^{\prime}$ su $\rho_{1}$ e $\rho_{2}$ rispettivamente: le proiezioni $V_{1}$ e $V_{2}$ di $V$ da $r^{\prime \prime}$ e $r^{\prime}$ su $\rho_{1}$ e $\rho_{2}$ dovranno appartenere a $f_{1}$ e $f_{2}$, e quindi $V_{1}$ e $V_{2}$ non possono essere delle trivarietà, ma al più delle tele, anzi tele curvilinee, cioè coincidenti con $f_{1}$ o $f_{2}$. In particolare $V_{1} \circ V_{2}$ (ma non entrambe) possono ridursi ad un filo (di $f_{1} \circ f_{2}$ rispettivamente). In ogni caso $V_{1}$ e $V_{2}$ prolungate nel corpo complesso, devono dare i due tratti di curve $f_{1}$ e $f_{2}$, cioè le funzioni $x_{1}, y_{1}\left(x_{2}, y_{2}\right)$ date dalle (V), che rappresentano in $\rho_{1}\left(\rho_{2}\right) V_{1}\left(V_{2}\right)$ prolungate nel corpo complesso, devono dare due funzioni complesse olomorfe di tre variabili complesse che devono ridursi $a$ funzioni olomorfe di una sola variabile complessa $\mathrm{z}_{1}\left(\mathrm{z}_{2}\right)$.

Viceversa, se queste condizioni sono soddisfatte, le proiezioni $V_{1}$ e $V_{2}$ della trivarietà $V$ apparterranno a due tratti di curve $f_{1}$ e $f_{2}$ e la $V$ appar- 
terrà alla superficie caratteristica $F$ rispondente a $f_{1}$ e $f_{2}$. Si ha pertanto il teorema:

II) Sia $\mathrm{V}_{\mathrm{x}}$ una varietò reale tridimensionale dell' $\mathrm{S}_{4}$ reale di equazioni $\left(V_{\mathrm{x}}\right)$ o $\mathrm{y}^{*}(\mathrm{x})$ una funzione bicomplessa del punto variabile su $V_{\mathrm{x}}$ data dalla (6).

Sia inoltre $\mathrm{V}$ la trivarietà dell' $\mathrm{S}_{4}$ complesso determinata da $\mathrm{V}_{\mathrm{x}}$ e da $\mathrm{y}^{*}(\mathrm{x})$. Condizione necessaria e sufficiente affinchè esista una funzione bicomplessa $\mathrm{y}(\mathrm{x})$ definita in un intorno quadridimensionale di $\mathrm{V}_{\mathrm{x}}$, che su $\mathrm{V}_{\mathrm{x}}$ si riduca alla $\mathrm{y}^{*}(\mathrm{x})$, e olomorfa secondo Sconza DRAGonI, o totalmente derivabile, è che le proie. zioni della trivarietà $\mathrm{V} d a \mathrm{r}^{\prime \prime}$ e $\mathrm{r}^{\prime}$ sui piani coordinati $\rho_{1}$ e $\rho_{2}$ siano curvilinee, ovvero che le prime (seconde) funsioni (V), prolungate nel campo complesso, si riducano a due funzioni olomorfe di una sola variabile complessa. Se queste condizioni sono soddisfatte, la funzione $\mathrm{y}(\mathrm{x})$ soddisfacente alle condizioni volute $\grave{e}$ unica. 